\title{
Demand Side Flexibility Prospects in Modern LV Networks: A Probabilistic Assessment
}

\author{
Iasonas I. Avramidis, Vasileios A. Evangelopoulos, and Pavlos S. Georgilakis \\ School of Electrical and Computer Engineering \\ National Technical University of Athens (NTUA) \\ Athens, Greece \\ jasonavra@yahoo.com, vevangel@power.ece.ntua.gr,pgeorg@power.ece.ntua.gr
}

\begin{abstract}
Modern and flexible schemes facilitating higher penetration of residential distributed energy resources (DERs), such as photovoltaics, wind generators, electric heat pumps and electric vehicles in low voltage (LV) networks are of high interest in recent years. This paper investigates multiple simplified demand side flexibility (DSF) schemes to achieve said goal, while considering uncertainties that are related to the stochastic behaviour of the various network components and DERs. A probabilistic assessment methodology is adopted based on the Monte Carlo simulation, whilst real LV feeders and DER profiles were used to measure the performance of the investigated DSF schemes. The results include a probabilistic assessment for the technical performance of each DSF scheme by means of power delivery, operational improvement in higher penetration levels of residential DERs, and a thorough comparison of the effectiveness between these DSF schemes.
\end{abstract}

Index Terms-Demand Side Flexibility, Distributed Energy Resources, Monte Carlo Simulation, Probabilistic Power Flow.

\section{INTRODUCTION}

Small-scale distributed energy resources (DERs), like roof-top photovoltaics (PVs), wind generators (WGs), electric heat pumps (EHPs) and electric vehicles (EVs) are experiencing a constantly increasing use in daily life by residential consumers. They can all be viewed as low carbon technologies, because of their nature to be renewable (e.g., PVs, WGs) or their trait of not emitting greenhouse gases when used in urban areas (e.g., EVs, EHPs). Promoted by governments and the energy industry, residential DERs are expected to highly penetrate in low voltage (LV) power distribution systems by 2030. Although modern DERs contribute to ecological footprint reduction, their inherent stochastic nature and their uncoordinated integration lead to technical issues and constrain the level up to which such technologies can penetrate in LV network problem-free. The uncertainties of residential DERs are related to their random interconnection in LV networks as well as to the shifting weather conditions that lead to uncontrollable power output.

Several papers have investigated the technical issues of residential DERs in LV networks, some without considering the factors of uncertainty related with these issues [1]-[2], and others having assessed their impact in terms of probabilistic analysis [3]-[5]. In [1], the technical issues due to increasing EVs penetration in LV networks are investigated, while in [2] the impact of residential roof-top PVs in LV networks is investigated for different penetration levels. The probabilistic analysis framework of residential DERs impact has been established in [3] for assessing the impact of EHPs in LV networks. The impact of different residential DER technologies is assessed in [4] under uncertainties in terms of location, size and behavior. Monte Carlo simulation (MCS) is used by [3]-[4] to outline the likelihood of technical issues. Conversely, the point estimate method is introduced in [5] to solve the probabilistic power flow problem for a single penetration level.

The above literature review highlights the need for new schemes that will facilitate the smooth operation of LV networks stressed with high penetration of residential DERs. This paper investigates several different demand side flexibility schemes (DSF) based on simplified, yet realistic, rules. These DSF schemes are proven to ensure problem-free power delivery to end-customers in high DER penetrations. The DSF schemes are based on demand side management (DSM) and demand response (DR) methods, coupled with specific intervention tool to increase, decrease or redistribute the total demand. This paper is focused on the performance of the DSF schemes, which is evaluated by a probabilistic assessment method based on MCS. The results extracted serve as a guide for distribution system operators (DSOs) and are indicative of the uncertainties that each DSF scheme has. The DSO can make its decisions depending on how low the probability of a technical issue is. Through this analysis, reinforcement and/or costly infrastructural investments to the distribution network (to ensure safe operation and reliability) may be postponed depending on how efficient and reliable the proposed DSF schemes are. In addition, the proper study and management of the probabilistic analysis may form the basis for rotating schemes between the DSF methods, based on economic factors, weather conditions and variability in the customers' willingness to participate in each scheme. 


\section{LOAD \& DER: PROFILES \& FEATURES}

This work utilizes large pools of daily 5-min resolution time series profiles of residential loads and DERs. DER types considered for the scope of this study are WGs, PVs, EHPs, and EVs. Each pool of profiles comprises of 2,000 profiles, augmented from pools originally consisting of 100 profiles, available in [6]. The mainstream behavior of each load type and DER, i.e. the average profiles, is illustrated in Fig. 1. Table I summarizes the most representative characteristics, i.e. peak and minimum power, daily energy, and daily time duration of demand/production over the $80 \%$ of peak power consumed/produced, respectively. The later corresponds to the time period when the most severe technical issues have the higher likelihood to occur.

a) Residential loads: 2,000 summer profiles and 2,000 winter profiles, artificially augmented from 100 profiles, under the assumption that residential loads largely follow the normal distribution in any distinct period.

b) WGs: Assuming that wind speed follows the Weibull distribution, 2,000 profiles were artificially created, considering residential WGs of $2 \mathrm{~kW}$ nominal power, with 4 $\mathrm{m} / \mathrm{s}$ cut-in wind speed, $15 \mathrm{~m} / \mathrm{s}$ nominal wind speed and 25 $\mathrm{m} / \mathrm{s}$ disconnection wind speed. Data were collected for the three windiest months of 2016 in the UK.

c) PVs: Utilizing the original 100 individual $\mathrm{PV}$ profiles as well as information on sun irradiance, efficiencies of energy conversion, and PV inverter from [4], 2,000 profiles were created under the assumption that PVs follow the Weibull distribution in any distinct period.

d) EHPS: 2,000 profiles were created similarly to how residential loads were produced, under the assumption that EHPs follow the very similar patterns of behavior and utilizing the 100 individual air source EHP profiles of [6].

e) EVs: In this case, the artificial augmentation to 2,000 profiles was more challenging, due to the differences between EVs and traditional loads, particularly the EV's binary nature. The same method that was applied in [4] is also used here, by considering the probability distribution of connection times and the required energy absorption.

\section{DSF SCHEMES}

Five different DSF schemes are investigated, which are employed to facilitate the higher penetration of residential DERs, each for different DER type. These schemes are:

a) Load shifting (LS): applied in the presence of energy-consuming DER, redistributing the energy demand but maintaining its size.

b) Reverse $L S(R L S)$ : applied in the presence of energyproducing DER, redistributing the energy supply but maintaining its size.

c) Strategic conservation (SC): applied in the presence of energy-consuming DER, decreasing the energy demand.

d) Strategic load growth (SLG): applied in the presence of energy-producing DER, increasing the energy demand.

e) Flexible load $(F L)$ : applied for both kinds of DER (energy-consuming and energy-producing), re-shaping the energy demand on a case-by-case basis.

\begin{tabular}{c|c|c|c|c|c}
\hline \multicolumn{2}{c|}{ Load \& DER types } & $\begin{array}{c}\text { Peak } \\
(\boldsymbol{k W})\end{array}$ & $\begin{array}{c}\text { Min } \\
(\boldsymbol{k W})\end{array}$ & $\begin{array}{c}\text { Daily Energy } \\
(\boldsymbol{k W h})\end{array}$ & $\begin{array}{c}\text { Time over 80\% of } \\
\text { Peak (min) }\end{array}$ \\
\hline \multirow{2}{*}{$\begin{array}{c}\text { Residential } \\
\text { Load }\end{array}$} & Summer & 0.86 & 0.07 & 9.03 & 95 \\
\cline { 2 - 6 } & Winter & 1.06 & 0.09 & 10.44 & 155 \\
\hline \multirow{2}{*}{$\begin{array}{c}\text { Producing } \\
\text { DER }\end{array}$} & WG & 0.73 & 0.41 & 13.03 & 365 \\
\cline { 2 - 6 } & PV & 3.10 & 0.00 & 20.67 & 160 \\
\hline $\begin{array}{c}\text { Consuming } \\
\text { DER }\end{array}$ & EHP & 2.24 & 0.03 & 19.82 & 95 \\
\cline { 2 - 6 } & EV & 1.23 & 0.00 & 8.32 & 110 \\
\hline
\end{tabular}

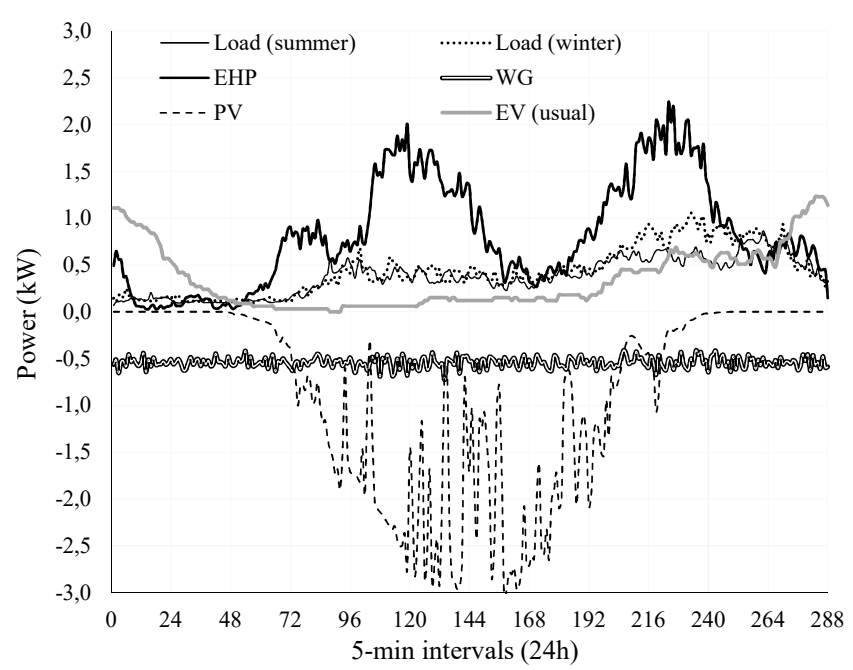

Figure 1. Average profiles of load and DERs under investigation.

The schemes may belong to one of two categories: a) coordinated but not optimized usage of the available DER in the network (LS, RLS, SLG), or b) optimized behavior of the individual customer by considering the needs of the whole network (FL, SC). The main goal is to reduce voltage problems (overvoltages/undervoltages) and thermal issues in the studied networks. What is most important is that the schemes are very effective while still unoptimized, and that they have wide customer acceptance because of their simplicity. This paper highlights the "raw strength" of each scheme, which, coupled with their respective customer opinion on them, serves as an excellent guideline on which schemes are worth further pursuing with respect to increasing the selected residential DER's penetration. Three key points should be stressed, regarding the assumptions, selection process and design approach:

1) Apart from the network equipment, the DSO owns no resources (true in several countries). The intervention tools utilized in each scheme (e.g., EVs, ESS) are 100\% customerowned, and their usage depends solely on the cooperation of the customer. The penetration of tools is equal to the penetration of residential DERs (spread randomly among customers though), as it is assumed that the growth trend in low-carbon energy and home energy management is similar.

2) Based on point 1 , the customers should be persuaded to consent to the DSO using their DER. A financial incentive is necessary; however, it is insufficient in many cases. The network's proper operation is not something that would inherently interest the customers. Additionally, most of them require a clear understanding of how their equipment is used. 
Thus, each scheme should be very intuitive and easy enough to understand. The schemes are thus designed with a very simple, yet effective, rule-based approach in mind, like for example, ESS charging occurring during midday and discharging during the night (correlated with regular PV behavior). Based on the schemes' simplicity, a conducted survey of over 700 people, which is briefly presented in Table II, highlights that the overwhelming majority of customers (over 90\%) are willing to accept them immediately.

3) All schemes are used in a rule-based form. They either involve no optimization, or the optimization is done percustomer and not network-wide. On average, it is also assumed that all devices studied are identical. This is only a convention; the design of schemes makes them very easily adaptable to any overall characteristics, and the same final conclusions can be drawn. Apart from simplicity, and thus high adoptability by customers, a significant advantage of our approach is that for each scheme, actual effectiveness and potential for further targeted research are easily estimated. The schemes deemed intuitively superior are proven to deem further optimization (while always keeping in mind that simplicity is associated with easier adoption). This approach aims to make a good estimate of the proper balance between simplicity and effectiveness. As will be displayed in Section $\mathrm{V}$, this does not hinder the schemes' potential; in most cases, the improvements in network operation are very significant.

Below is a brief description of the schemes utilized:

a) $L S$ and RLS: The schemes are based on shifting surplus demand (LS) or supply (RLS) to time periods less stressful on the grid. This is realized with the use of a percase charging schedule involving commercially available residential ESS equipment. Information in Table III refers to the preprogrammed operation of ESSs when used with PVs, EHPs or EVs, accordingly. To ensure a longer lifetime for the ESS devices, one cycle of charging/discharging is executed each day, and the initial state of charge (SoC) is designed to be equal to the final $\mathrm{SoC}$ over the scheduling horizon. While sometimes these schemes fall in unavoidable pitfals (e.g. energy absorption while PVs are not producing or partially producing and vice versa), they are on average responsible for the redistribution of $35 \%$ to $70 \%$ of circulating energy, having a tremendous impact on regulating originaly arising issues. These schemes would require ESSs to grow along with the other residential DERs.

b) SC: The scheme combines environmentalsensitivity-driven reduction of energy needs and more efficient energy use (improved load management). Five predetermined strategies are chosen and optimized internally (HVAC equipment schedule, zone temperature set-point, chilled/condenser water set-point, supply air set-point, and human centric lighting schedule) to reduce total energy as much as possible. These are all built in and evaluated using Demand Response Quick Assessment Tool of U.C. Berkeley Lab [7]. The application of any and all measures happens solely at the customer level. Table II indicates that customers prefer managing their energy demand for financial incentives instead of "consiously" reducing their demand. Each customer randomly follows one to five of the strategies, and the calculation of the new total 3-month power profile is

\section{TABLE II. CONSUMERS RESPONSE RATE TO DSF SCHEMES}

\begin{tabular}{c|c|c|c|c}
\hline \multirow{2}{*}{ Question } & \multicolumn{4}{|c}{$\begin{array}{l}\text { "Would you accept the proposed DSF scheme, if offered } \\
\text { adequate financial leverage?" }\end{array}$} \\
\hline Answers & 760 answers, people ages 17-70, average age 51 years \\
\hline DSF Scheme & Intervention tool & Yes (\%) & No (\%) & Other (\%) \\
\hline \multirow{2}{*}{ SLG } & EVs (Weekdays) & 85.3 & 11.1 & 3.6 \\
\cline { 2 - 5 } & EVs (Weekend) & 95.6 & 0.8 & 3.6 \\
\hline LS, RLS & Residential ESS & 97.5 & 1.5 & 1.0 \\
\hline \multirow{2}{*}{ SC } & Demand Reduction & 5.0 & 94.5 & 0.5 \\
\cline { 2 - 5 } & Load Management & 98.5 & 1.0 & 0.5 \\
\hline FL & Dynamic Pricing & 81.0 & 11.0 & 8.0 \\
\hline
\end{tabular}

TABLE III. ESS CHARACTERISTICS FOR DSF SCHEMES

\begin{tabular}{c|c|c|c|c|c|c|c}
\hline DER & $\begin{array}{c}\text { DSF } \\
\text { Scheme }\end{array}$ & $\begin{array}{c}\text { Rated } \\
\text { Power } \\
(\boldsymbol{k W})\end{array}$ & $\begin{array}{c}\text { Capacity } \\
(\boldsymbol{k W h})\end{array}$ & $\begin{array}{c}\boldsymbol{C R}^{1} \\
(\boldsymbol{k W})\end{array}$ & $\begin{array}{c}\text { DR } \\
(\boldsymbol{k W})\end{array}$ & $\begin{array}{c}\text { Charging } \\
\text { period }\end{array}$ & $\begin{array}{c}\text { Discharging } \\
\text { period }\end{array}$ \\
\hline PV & RLS & 5.0 & 13.5 & 1.10 & 0.93 & $06: 00-17: 00$ & $17: 00-06: 00$ \\
\hline EHP & LS & 5.0 & 13.5 & 1.00 & 1.00 & $00: 00-08: 00$ & $\begin{array}{c}08: 00-11: 00 \\
16: 00-21: 00\end{array}$ \\
\hline EV & LS & 5.0 & 13.5 & 0.375 & 0.75 & $17: 00-01: 00$ & $01: 00-17: 00$ \\
\hline
\end{tabular}

${ }^{1} \mathrm{CR}=$ Charging rate, ${ }^{2} \mathrm{DR}=$ Discharging rate.

executed profile (similar in form but reduced energy-wise). Depending on the DER used in conjunction with, the scheme may achieve a total load reduction of $5 \%$ to $15 \%$ (EHP), or of $1 \%$ to $6 \%(\mathrm{EV})$. The differences can be attributed to the fact that the profile of EVs cannot be inherently affected by such a scheme. This scheme is easily implementable and can be achieved by a home energy management system (HEMS).

c) SLG: Through coordinated EV charging, the scheme achieves artificial augmentation of customer demand, to combat issues associated with excessive DER power production. Two EV charging profiles, the daily average usual (Fig. 1) and the daily average desired [8], are used to synthesize the daily average final. However, it is assumed that people need to regularly use EVs during weekdays. Thus, the scheme separates the participants into five equal groups, each applying the desired charging plan during a different weekday (i.e. group 1 on Monday, group 2 on Tuesday, etc.), while everyone is a willing participant during the weekends. Thus, for an average day, $39.5 \%$ of users fully adopt the desired charging profile, $8 \%$ of users follow their own charging schedule (Fig. 1), while the remaining $48.8 \%$ of users do not charge their EVs at all [8]. In its totality, the charging scheme combines two problem-ridden residential DERs and manages to cancel out about $55 \%$ of total circulating energy. This scheme would require EVs to grow along with PVs, an approach that has already garnerned significant research attention, producing many optimal coordinations of EV-PV combinations in a grid.

d) FL: At any given time, this scheme may increase, decrease, or maintain the demand profile. Thus, the daily profile is subject to a case-by-case type of change, according to the network's needs. Through intensive dynamic pricing, the hourly energy price is heavily altered, with the aim to create a smoother demand profile. When the expected overall power profile is overly negative, prices are sharply lowered to encourage consumption and vice versa. Here, a deterministic version of the DR model developed in [9], is used. This is the only scheme that is applicable for every single DER. 
However, while the scheme is applied based on the overall load, only the residential load profiles and EHP profile can be meaningfully affected. PVs are obviously unaffected, while EVs are assumed to remain largely unaffected, due to the inflexibility between their two states (charge/no charge) and because they are assumed to have a full single charge per day, which would be stressful for the grid regardless of the time. The hourly energy prices used for winter and summer are the per-hour winter-average and summer-average wholesale UK energy price for 2016. This scheme would require customers being part of a dynamic DR system; this is also easily implementable by using a HEMS (assuming the DSO is also properly equiped; already a reality in many places).

\section{PROBABILISTIC Assessment Methodology}

To capture the impact of each DER and evaluate the performance of each DSF scheme, each DER is examined separately for the worst-case scenario, i.e. when the weather conditions are such that it would put the highest stress on the network. In that regard, WG and EHP profiles correspond to winter conditions (winter load), PV profiles correspond to summer conditions (summer load), and EV profiles correspond to both winter and summer conditions.

\section{A. Methodology}

For the probabilistic assessment of the investigated DSF schemes, an MCS-based stochastic power flow analysis algorithm was employed, originally developed in [4]. It considers daily time-series of 5-min intervals and is executed using the OpenDSS tool [10]. The uncertainties that are taken into consideration are related not only to the varying load and DER power, but also to the location and customer behavior. Given that the penetration level is defined as the percentage of customers owning and utilizing a DER, different penetration levels were selected from 0 to $100 \%$, with step of $10 \%$.

The methodology followed is briefly outlined as follows:

i. Initialization (Define feeder and DER studied)

ii. Set $n$ (number of customers of the chosen feeder)

iii. Set $p$ (DER penetration level \%)

iv. For each MCS iteration (Start MCS)

a. Randomly select $\mathrm{n}$ load profiles

Randomly allocate them to $\mathrm{n}$ customers

If (DSF scheme $=\mathrm{SC}$ or FL)

modify the load profiles (Section III.b or III.d)

b. Randomly select $\mathrm{n} \times \mathrm{p}$ DER profiles and allocate them to $n \times p$ random customers

If $($ DSF scheme $=$ SLG $)$ modify the DER profiles (Section III.c)

else If (DSF scheme $=$ LS or RLS)

apply the ESS charging schedule (Section III.a)

c. Execute the power flow analysis and obtain results per simulation

v. Gather the results of all simulations and calculate the performance metrics presented in Section IV.B

\section{B. DER Impact \& DSF Performance}

To quantify the impact of DERs as well as the performance of the DSF schemes, the percentage of customers with voltage problems according to EN 50160
TABLE IV. MAIN CHARACTERISTICS OF LV FEEDERS

\begin{tabular}{c|c|c|c|c|c|c|c|c|c}
\hline \multirow{2}{*}{$\begin{array}{c}\boldsymbol{L} V \\
\text { feeder }\end{array}$} & \multirow{2}{*}{$\begin{array}{c}\text { Length } \\
(\boldsymbol{m})\end{array}$} & $\begin{array}{c}\text { Custom. } \\
\text { Nr. }\end{array}$ & \multicolumn{2}{|c|}{$\begin{array}{c}\text { Winter } \\
\text { Load }(\boldsymbol{k W})\end{array}$} & \multicolumn{2}{c}{$\begin{array}{c}\text { Summer } \\
\text { Load }(\boldsymbol{k W})\end{array}$} & \multicolumn{4}{c}{$\begin{array}{c}\text { Connections (\%) } \\
\text { per feeder phase }\end{array}$} \\
\cline { 5 - 11 } & & & peak & $\min$ & peak & min & $\boldsymbol{R}$ & $\boldsymbol{S}$ & $\boldsymbol{T}$ \\
\hline N2F5 & 734.9 & 23 & 24.3 & 2.1 & 19.7 & 1.5 & 39.13 & 34.78 & 26.09 \\
\hline N1F1 & 1437.8 & 55 & 58.1 & 5.0 & 47.1 & 3.6 & 38.18 & 34.55 & 27.27 \\
\hline N2F3 & 2763.6 & 112 & 118.3 & 10.2 & 95.9 & 7.4 & 37.5 & 30.36 & 32.14 \\
\hline N2F1 & 5205.6 & 175 & 184.9 & 16.0 & 149.9 & 11.6 & 34.86 & 34.28 & 30.86 \\
\hline
\end{tabular}

(CVP) is computed. This metric expresses the expected number of customers not supplied with power over a day. The CVP, presented as percentage, is the average of customers having a voltage problem over the result of the MCS divided by the total number of customers in the feeder [4]. According to EN 50160 standard [11], the rms voltage value across the feeder shall be within the range from $-15 \%$ to $+10 \%$, i.e. 0.85 per unit (pu) to $1.1 \mathrm{pu}$. Additionally, only 5\% of 5-min voltage values being below $-10 \%$ is accepted over a day.

\section{Probabilistic Analysis}

The probabilistic analysis is based on the MCS results. The statistics extracted is the CVP average value plus and minus the standard deviation, as well as the cumulative distribution function (CDF) per DER type, DSF scheme (if applied) and penetration level.

The CVP average values give an estimation of the voltage problems expected to occur, whilst their standard deviation is related to the average distance the results have from the expected value. The higher the standard deviation is, the higher the uncertainty of the expected values is.

The CDF, in turn, is presented as a statistical curve and is employed to quantify the probability of having at least a certain percentage $x \%$ of problems. More specifically, the CDF gives the probability of appearance at least $x \%$ of CVP. While many metrics were introduced in [7], the CVP and CDF are the most important [4], and thus displayed here.

\section{CASE STUdy \& Results}

\section{A. Case Study}

The DER impact and the performance of the investigated DSF schemes are assessed, based on the methodology of Section IV, in four real UK LV feeders, the data of which are available in [6]. Table IV briefly presents the number of customers and the main characteristics per feeder. All LV feeders operate with nominal phase voltage of $230 \mathrm{~V}$ and their loads are supplied by a MV/LV 11/0.4 kV, $750 \mathrm{kVA}$ transformer. The LV side of the transformer is set at $1.05 \mathrm{pu}$ (241.5 V phase voltage) to avoid severe voltage drops along the feeder during periods of peak load. The power factor is considered 0.95 lagging for all loads and DERs. The load profiles and the residential DER profiles connected on the LV feeders under investigation are these described in Section II.

Firstly, each LV feeder is tested per DER type without applying any DSF scheme. The scope is to highlight the level of the DER-related problems. Afterwards, each proposed DSF scheme per DER type is employed to aid the improvement of power quality in higher penetration levels of DERs. 


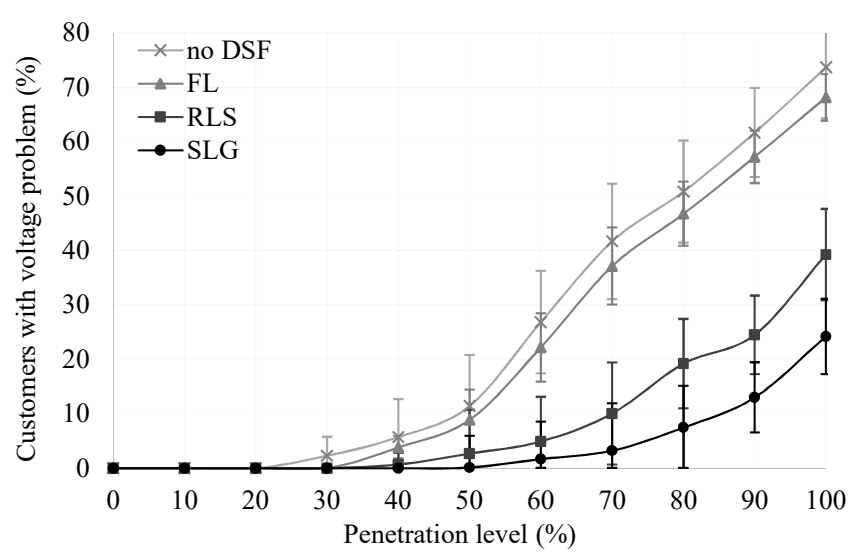

(a)

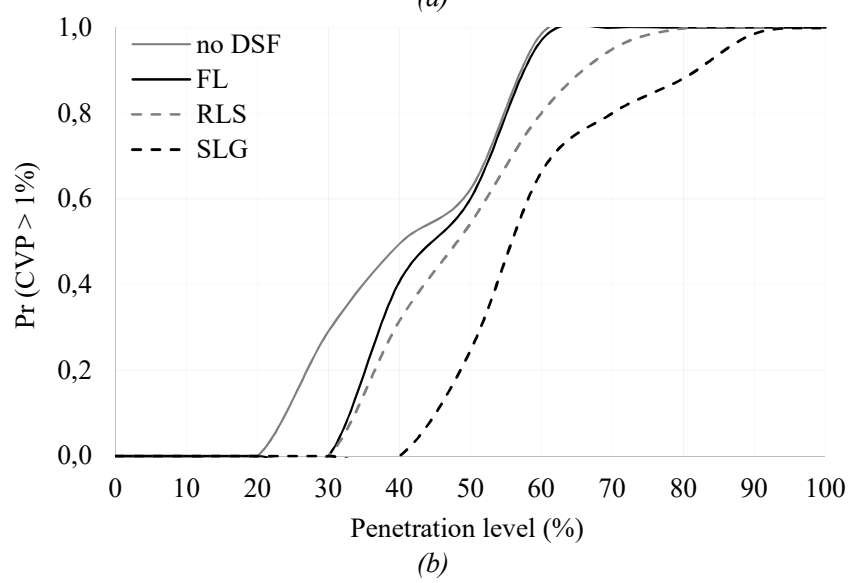

Figure 2. PV case: a) Average value \pm standard deviation of CVP, b) Probability of having at least $1 \%$ of CVP.

The methodology of Section IV was applied for all feeders and for each DER type. As was expected, the larger the feeder is, the more power quality problems exist, and thus the results obtained for the feeder N2F1 are analyzed hereafter.

\section{B. Results and Discussion}

Given that each DER type is tested independently, the discussion over the results is distinguished per DER type. The operation of small-scale WGs does not impose any problem to the feeders under examination, even for the highest penetration level. Therefore, the interest of this analysis is focused on the remaining DER types, i.e. PVs, EHPs, EVs.

a) PVs: PVs were studied during summer days, when solar irradiance takes its highest values. Fig. 2a depicts the expected impact of PVs installed in the tested N2F1 feeder and also illustrates the performance of the DSF schemes proposed to cater for PV penetration. SLG is more effective than FL and RLS, resulting in much lower CVP for the same penetration levels. For example, when PVs are hosted by $50 \%$ of the feeder's customers, more than $10 \%$ of customers suffer from overvoltage issues. By applying the SLG scheme, these technical issues can be eliminated and the feeder can operate problem-free even with $50 \%$ of the customers utilizing a PV.

Although the voltage problems seem to have almost been eliminated at the $50 \%$ penetration level with SLG scheme in effect, Fig. $2 b$ indicates that there is 0.25 probability of having at least one customer with voltage problems. For sure,

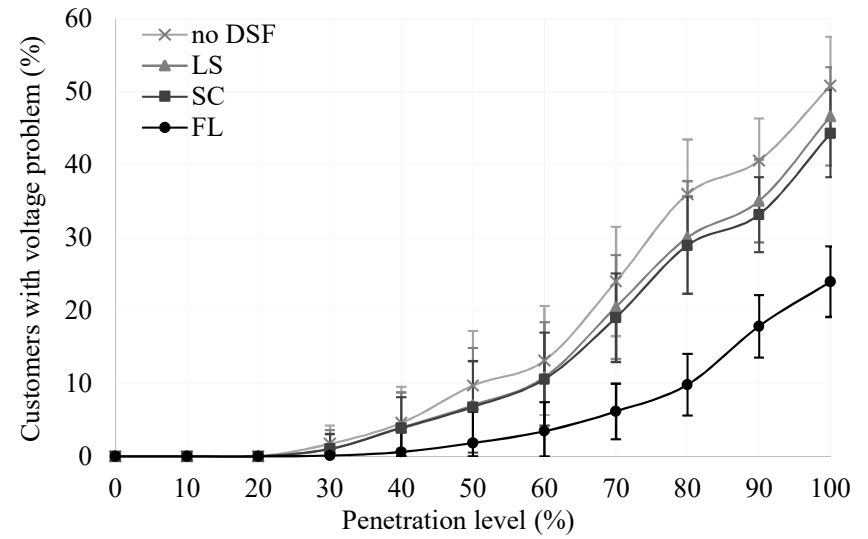

(a)

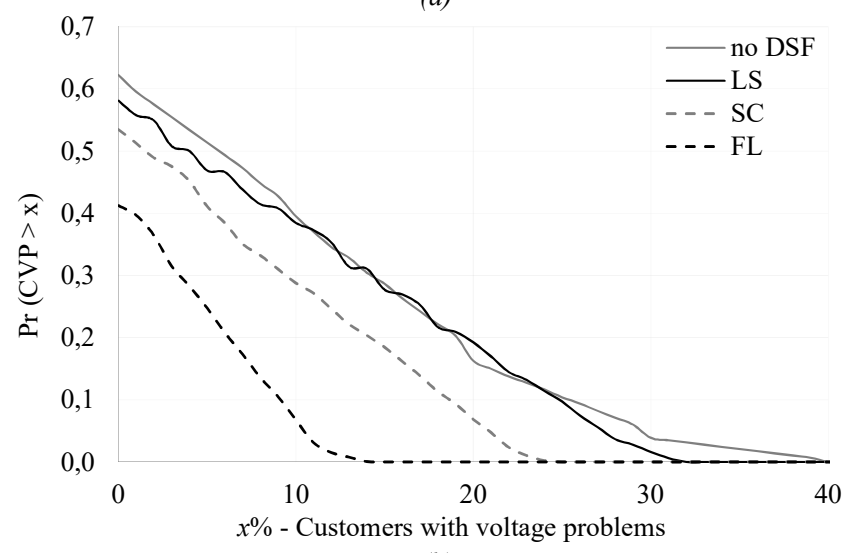

(b)

Figure 3. EHP case: a) Average value \pm standard deviation of CVP, b) CDF of CVP for $50 \%$ penetration level.

this is much better compared to the case where no DSF scheme is applied, yielding a probability of 0.62 , but highlights the importance of this probabilistic assessment. In total, the SLG scheme reduces voltage problems by up to $50 \%$.

b) EHPs: EHPs were studied during winter days, when they have the higher usage. The expected issue in this case is undervoltage along the feeder. Fig. 3a shows that problems begin at penetration level of $30 \%$, with the CVP increasing up to $51 \%$ for the $100 \%$ penetration level. As far as the DSF schemes for EHPs are concerned, LS and SC slightly improve the CVP metric, whilst FL decreases the problems and retains them in much lower levels even for higher penetration levels. FL not only mitigates the voltage related problems, but also gives results with low deviation from average. This makes FL more effective and robust when it comes to EHP in comparison with FL's performance for PV penetration.

Considering again penetration level $50 \%$ for EHPs, the probability of having at least $1 \%$ of customers with voltage problems is about 0.62 . This probability decreases down to 0.41 , when the FL is applied. From the findings depicted in Fig. 3b, one can highlight that FL decreases the probability of CVP over a certain percentage. EHP-related issues are drastically reduced through the FL scheme, by achieving a reduction of almost $50 \%$ on every metric and by reducing the probabilities of crossing set thresholds by up to $40 \%$.

c) EVs: In general, EVs cause much less voltage problems along the feeder. However, Fig. 4a highlights the 
high deviation the MCS results have from the mean values per penetration level. While on average voltage problems (CVP) firstly appear when over $30 \%$ of customers own an EV and gradualy increase to about $12 \%$ for maximum penetration, the full range of the issues and the accompanying uncerainties are very large, with the standard deviation of CVP going as high as $125 \%$ of the mean value. This is attributed to the high power demand of EVs and their binary, discrete nature as loads.

Fig. $4 \mathrm{~b}$ presents the probability curve for at least $1 \%$ of CVP per penetration level. LS scheme achieves almost $0 \%$ CVP at $70 \%$ penetration, i.e. the feeder is expected to operate almost without any voltage violation for DER penetration level up to $70 \%$. One notices that although LS seems to have better performance than FL when considering the average values from the MCS results, Fig. 4b shows that FL has lower probability of at least $1 \%$ of voltage problems than LS has for penetration levels from $50 \%$ to $80 \%$. The LS scheme, in turn, has lower probability for penetration greater than $90 \%$. Interestingly, at certain penetration levels, it is slightly less effective than the FL scheme, due to the lower deviations that are achieved through FL due to heavy load manipulation. Concluding the EVs' results, the LS scheme achieves a doubling of acceptable EV penetration.

\section{CONCLUSION}

This paper examines the issues associated with the high penetration of several DER in residential LV networks, and offers several solutions in the form of different DSF schemes to combat said technical problems. Every single scheme achieves improvements in power quality and energy cost savings. In summary, the maximum problem-free penetration level is increased between $10 \%$ and $40 \%$, while voltage violations are significantly reduced. The probabilities of encountering voltage problems and of crossing the $1 \%$ of customers' threshold have been greatly diminished, with drops as large as $70 \%$. What the best approach is depends on the DER and the penetration level. On average, the CVP improvement (expressing customer satisfaction) is about $70 \%$, the probability of encountering issues at originally unacceptable penetration levels is reduced by up to $60 \%$, while the probability of crossing the $1 \%$ of customers with voltage violations is reduced by almost $25 \%$.

If one considers the heuristic way on which the schemes are designed (simplicity), their realistic design without excessive additional infrastructure investment (applicability) and their theoretically easily made mainstream applicability (public acceptance), this paper provides an initial evaluation for the DSF schemes. Those schemes that are deemed superior (per case) may be further optimized and stressed to their absolute limits. Of course, any and all such studies should always consider the three main axes of these DSF schemes: simplicity, applicability and public acceptance.

\section{REFERENCES}

[1] P. Richardson, D. Flynn, and A. Keane, "Impact assessment of varying penetrations of electric vehicles on low voltage distribution systems," in Proc. 2010 IEEE PES General Meeting, Providence, USA, Jul. 2010.

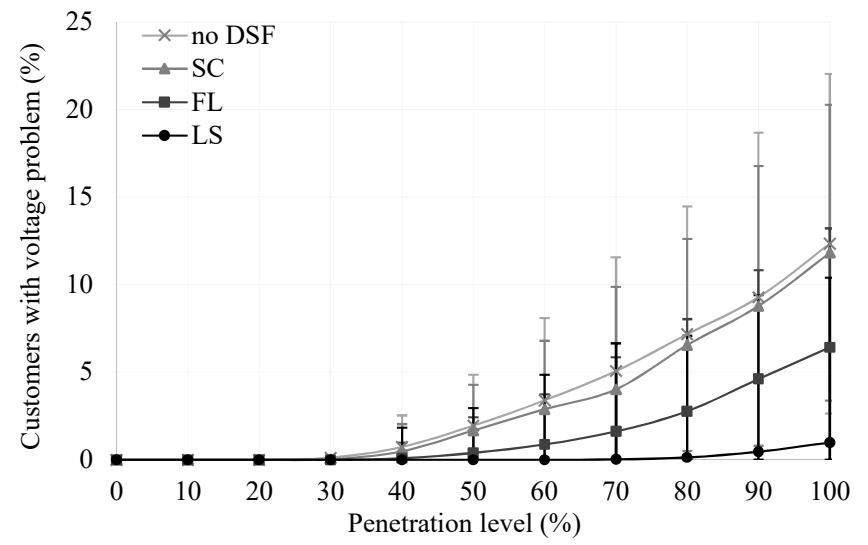

(a)

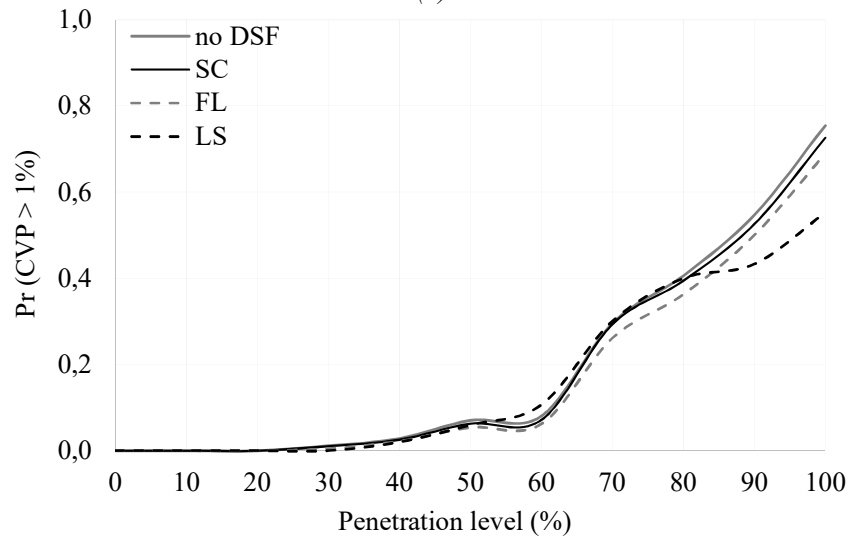

(b)

Figure 4. EV case: a) Average value \pm standard deviation of CVP, b) Probability of having at least $1 \%$ of CVP.

[2] J.D. Watson, N.R. Watson, D. Santos-Martion, A.R. Wood, S. Lemon, and A.J.V. Miller, "Impact of solar photovoltaics on the low-voltage distribution network in New Zealand," IET Gener. Transm. Distrib., vol. 10, no 1, pp. 1-9, 2016.

[3] A. Navarro-Espinosa and P. Mancarella, "Probabilistic modeling and assessment of the impact of electric heat pumps on low voltage distribution networks," Appl. Energy, vol. 127, pp. 249-266, Aug. 2014.

[4] A. Navarro-Espinosa and L.F. Ochoa, "Probabilistic impact assessment of low carbon technologies in LV distribution systems," IEEE Trans. Power Syst., vol. 31, no 3, pp. 2192-2203, May 2016.

[5] F. Diop and M. Hennebel, "Probabilistic load flow methods to estimate impacts of distributed generators on a LV unbalanced distribution grid," in Proc. 2017 IEEE PES PowerTech, Manchester, UK, 2017.

[6] ENWL: "Dissemination document - low voltage networks models and low carbon technology profiles," June 2015. [Online]. Available: https://www.enwl.co.uk/innovation/smaller-projects/low-carbonnetworks-fund/low-voltage-network-solutions

[7] Demand Response Research Center: "Demand response quick assessment tool" [Online]. Available: https://drrc.lbl.gov/tools

[8] I.I. Avramidis, V.A. Evangelopoulos, P.S. Georgilakis, and N.D. Hatziargyriou, "Demand side flexibility schemes for facilitating the high penetration of residential distributed energy resources," IET Gener. Transm. Distrib., vol. 12, pp. 4079-4088, Oct. 2018.

[9] A.J. Conejo, J.M. Morales, and L. Baringo, "Real-time demand response model," IEEE Trans. Smart Grid, vol. 1, no. 3, pp. 236-242, Dec. 2010.

[10] R. Dugan and T. McDermott, "An open source platform for collaborating on smart grid research," in Proc. 2011 IEEE PES General Meeting, Detroit, USA, Jul. 2011.

[11] EN 50160, Voltage characteristics of electricity supplied by public distribution systems, EN standard, 2007. 